\title{
Photoinduced intramolecular charge-transfer reactions in 4-amino-3- methyl benzoic acid methyl ester: A fluorescence study in condensed- phase and jet-cooled molecular beams
}

\author{
AMRITA CHAKRABORTY, ${ }^{a}$ SAMIRAN KAR,${ }^{\mathrm{a}, \mathrm{c}} \mathrm{D}^{\mathrm{N}} \mathrm{NATH}^{\mathrm{b}}$ and NIKHIL GUCHHAIT ${ }^{\mathrm{a}, *}$ \\ ${ }^{a}$ Department of Chemistry, University of Calcutta, 92, A.P.C. Road, Kolkata 700009 \\ ${ }^{b}$ Department of Physical Chemistry, Indian Association for the Cultivation of Science, Jadavpur, \\ Kolkata 700032 \\ ${ }^{c}$ Present address: CHEMGEN Pharma International, Dr. Siemens Street, Bolck GP, Sector V, \\ Salt Lake City, Kolkata 700091 \\ e-mail: nguchhait $@$ yahoo.com
}

\begin{abstract}
Photoinduced intramolecular charge-transfer reactions in 4-amino-3-methyl benzoic acid methyl ester (AMBME) have been investigated spectroscopically. AMBME, with its weak charge donor primary amino group, shows dual emission in polar solvents. Absorption and emission measurements in the condensed phase support the premise that the short wavelength emission band corresponds to local emission and the long wavelength emission band to the charge transfer emission. Laser-induced fluorescence excitation spectra show the presence of two low-energy conformers in jet-cooled molecular beams. Theoretical calculations using density functional theory help to determine structure, vibrational modes, potential energy surface, transition energy and oscillator strength for correlating experimental findings with theoretical results.
\end{abstract}

Keywords. 4-Amino-3-methyl benzoic acid methyl ester; fluorescence; quantum yield; laser-induced fluorescence; density functional theory; supersonic jet.

\section{Introduction}

Since the first observation of dual fluorescence from 4-N,N-dimethylamino benzonitrile (DMABN) by Lippert et al, ${ }^{1}$ spectral studies of donor-acceptor charge-transfer molecules have been fascinating to researchers both from the fundamental and application points of view. Upon photoexcitation in the UV region in suitable polar solvent DMABN shows fluorescence both in locally excited (LE) and charge transfer (CT) states. The higher energy, solventindependent emission band is assigned to emission from a benzenoid type $\pi-\pi^{*}$ LE state and the lower energy, highly solvent-dependent, red-shifted emission band arises from a highly polar intramolecular CT state. Studies on this type of photophysical phenomenon for donor-acceptor systems are important for understanding the primary processes of vision and photosynthesis, where charge transfers play key roles. Therefore, research has been going on with a variety of new donor-acceptor charge-transfer systems and there are various models proposed to interpret the entire mechanism of photoinduced intramolecu-

*For correspondence lar charge transfer in DMABN and related molecular systems. ${ }^{2-7}$ Briefly, in the twisted intramolecular charge transfer (TICT) model proposed by Grabowski et $a l^{4}{ }^{4}$ the initially generated LE state yields another minimum on the excited state potential energy surface by the twisting of the donor group into a plane perpendicular to the acceptor group, leading to a red-shifted CT emission. In the planar intramolecular charge-transfer (PICT) model, a solvent-induced vibronic coupling is supposed to take place between the LE and CT states leading to formation of a final planar structure. ${ }^{5}$ Apart from these two models, a rehybridized intramolecular charge transfer (RICT) model was proposed by Domcke and coworkers. ${ }^{6}$ None of these proposed models fully enable provision of a unified interpretation of the ICT process in donor-acceptor charge transfer systems. To date, the most accepted one is the TICT model. ${ }^{4}$ However, it is clear that dual fluorescence depends on the relative energies of the first two excited states, and solvents of different polarities fine tuned the energy gap between the states in different ways in different systems. Although DMABN shows dual fluorescence in polar solvents, it is well established experimentally and theoreti- 
cally that its primary amino derivative 4-aminobenzonitrile (ABN) does not show dual fluorescence even in highly polar solvent due to the large energy gap between the interacting excited states. From comparison of the spectral data of DMABN and $\mathrm{ABN}$, Zachariasse et $a l^{8}$ proposed that with the reduction of donor strength the energy gap between the first and second excited states becomes high, solvent cannot fine tune the large energy gap and hence dual fluorescence is not observed in $A B N$. Absence of dual emission in $\mathrm{ABN}$ may be the reason why most of the molecules so far studied for intramolecular charge-transfer process have tertiary amine (e.g. N,N-dimethylamine) groups as charge donors. There are very few examples where a primary amino group is used as the charge donor. Zachariasse and his group ${ }^{9}$ demonstrated ICT in some fluoro-substituted amino benzonitrile derivative and very recently Stalin et $a l^{10-12}$ reported charge-transfer reaction in $p$-amino benzoic acid, 3amino benzoic acid and 2-amino benzoic acid in the solution phase. There have been several attempts to investigate the same photo-induced CT process of bare molecules or their size-selected solvated clusters in jet-cooled molecular beams to obtain better understanding of the process. ${ }^{13-16}$ It is reported that ethyl and methyl- $p$-amino benzoate do not show dual fluorescence in the gas phase. ${ }^{17-19}$ Yonaggang's group ${ }^{20-22}$ have studied the gas phase water complex of $p$ - and $o$-amino benzoic acid using resonanceenhanced multiphoton ionisation and assigned the excited state vibrational modes. In this paper, we present the fluorescence spectroscopy of 4-amino-3methyl benzoic acid methyl ester (AMBME) in the condensed phase and in jet-cooled molecular beams. We also perform quantum chemical calculations to predict structure and photophysical properties of AMBME at density functional theory (DFT) level. With regard to the TICT model, twisting of the donor side has been considered a variable parameter for determining the ground- and excited-state potential energy surface. Solvation calculation has been performed using time-dependent density functional theory-polarizable continuum model (TDDFTPCM) to correlate some of the experimental spectroscopic findings with the theoretical results.

\section{Experimental section}

\subsection{Methods}

For the synthesis of 4-amino-3-methyl benzoic acid methyl ester (AMBME), $\mathrm{SOCl}_{2}(3.52 \mathrm{ml})$ was added dropwise to a solution of 4-amino-3-methyl-benzoic acid (Aldrich make) $(6 \mathrm{~g}, 40.3 \mathrm{mmol})$ in $\mathrm{MeOH}$ $(50 \mathrm{ml})$ under ice-cooled condition. The reaction mixture was stirred for $24 \mathrm{~h}$ at room temperature. The excess volatile solvent was removed under reduced pressure and the residue was dissolved in water, neutralized with aqueous $\mathrm{NaHCO}_{3}$ and extracted with ethyl acetate $(50 \mathrm{ml} \times 3)$. The combined organic layer was washed with water and brine, and then dried over anhydrous $\mathrm{Na}_{2} \mathrm{SO}_{4}$ the solvent was removed under reduced pressure to get the desired compound as a colourless solid $(6.3 \mathrm{~g}, 95 \%)$. The product was recrystallized to obtain the pure compound. ${ }^{1} \mathrm{HNMR}\left(\mathrm{CDCl}_{3}, 60 \mathrm{MHz}\right) \delta 2 \cdot 1(s, 3 \mathrm{H}), 3 \cdot 7$ (brs, 2H), $3.95(s, 3 \mathrm{H}), 7.6-7.9(m, 3 \mathrm{H})$.

Spectroscopic-grade solvents from SpectrochemUV and ethanol from E Merck were used after vacuum distillation. All solvents were checked for any fluorescence in the desired wavelength region before performing any emission studies. Sulphuric acid from E Merck was used as supplied. Triple distilled water was used for making all aqueous solutions.

\subsection{Steady-state and time-resolved spectral measurements}

Absorption and emission spectra of AMBME were recorded on a Hitachi UV/VIS U-3501 spectrophotometer and Perkin-Elmer LS50B fluorimeter, respectively. All spectral measurements were done at $\sim 10^{-6} \mathrm{M}$ concentration of solute in order to avoid aggregation and self-quenching.

The laser system used for time-resolved experiments consists of a Coherent Mira 900F femtosecond laser system, the output of which was pulsed picked (Coherent 9200 pulse picker) at a rate of $3.8 \mathrm{MHz}$ and then frequency-tripled in an ultrafast harmonic generation system (INRAD 5-050). Fluorescence decays were recorded using a time-correlated singlephoton counting (TCSPC) system from Edinburgh Instruments (Lifespec-Red). This system has an instrument response function (IRF) of $180 \mathrm{ps}$. The fluorescence decays were deconvoluted using the iterative software provided by the manufacturer. ${ }^{23}$

\subsection{Laser-induced fluorescence (LIF) excitation measurement}

The experimental setup for the measurement of LIF excitation spectrum in jet-cooled molecular beams has been newly built in our department. In brief, the 
sample vapour of the title compound seeded in helium gas was expanded from high pressure $(\approx 1 \mathrm{~atm})$ to vacuum $\left(<10^{-4}\right.$ Torr $)$ through a home-made pulsed nozzle valve. The pulsed valve has been made with a fuel injector and is controlled by a home-made pulsed valve driver. The sample was heated to $\approx 100^{\circ} \mathrm{C}$ to get enough vapour pressure. A Nd : YAG (Quanta System) pumped dye laser (Lambda Physik, FL 2002) has been used to generate tunable visible light. The visible dye laser output is frequency doubled by a KDP crystal and is introduced to the vacuum chamber perpendicular to the molecular beam direction for exciting the isolated jet-cooled molecules. Fluorescence signals from the samples have been collected perpendicular to both the exciting light and the molecular beam by a two-lens collecting system and detected by a photomultiplier (IP 28). The output of the photomultiplier has been averaged by a home-made Boxcar Integrator and finally processed using a chart recorder and personal computer.

\subsection{Computational details}

All structural and potential energy surface calculations were performed using Gaussian 03 package. ${ }^{24}$ The ground-state low-energy conformers of AMBME have been optimised with B3LYP hybrid functional and $6-311++\mathrm{g}(d, p)$ basis sets. Calculated vibrational frequencies for each conformer for the ground state have been done with the same functional and basis set. Excitation energies were calculated using TDDFT method implemented in Gaussian using the same functional and basis sets. ${ }^{25}$ Computed excitation energies are the vertical transition energies without zero point correction. We also extended our calculations in solvated systems using a non-equilibrium TDDFT-PCM model. ${ }^{26}$ Calculations of the potential energy surface (PES) were pursued along the twist coordinate at the donor $\left(-\mathrm{NH}_{2}\right)$ and acceptor $(-\mathrm{COOMe})$ side separately. One limitation is that the above-calculated PES only corresponds to the cut-off PE-hyper surface along the twisting angle as no geometry optimisation of the various excited states has been performed. This approach, i.e., the use of ground-state optimised geometry as a basis for the representation of the excited-state structure, has been successfully applied in many recent studies on donor-acceptor charge-transfer reactions. ${ }^{27-30}$

\section{Results and discussion}

The absorption and fluorescence spectra of AMBME have been studied in solvents of different polarities and the experimental results are presented in table 1. The molecule AMBME shows a strong structureless broad absorption band with $\lambda_{\max }$ at $\approx 280 \mathrm{~nm}$. This absorption band is basically the $\pi-\pi^{*}$ type of transition as was observed in case of benzene-like systems. ${ }^{10-12}$ As seen in figure la, polarity of the solvent has very little effect on the absorption peak position, indicating the weak polar character of the system in the ground state. Comparing the absorption spectra with other related systems, this broad band indicates a possible overlap of $S_{1}$ and $S_{2}$ states in AMBME ${ }^{8}$ Later on we have found that the calculated energy difference between $S_{1}$ and $S_{2}$ states is not very high.

The emission spectra of AMBME were recorded in different solvents by excitation at $280 \mathrm{~nm}$. As

Table 1. Spectroscopic parameters obtained from absorption and emission band maxima of 4-amino-3-methyl benzoic acid methyl ester in different solvents.

\begin{tabular}{|c|c|c|c|c|c|c|c|}
\hline Solvents & $\lambda_{\mathrm{abs}}(\mathrm{nm})$ & $\lambda_{\text {flu }}(\mathrm{nm})$ & $\phi$ & $\Delta v$ & $\tau_{1}\left(a_{1}\right)$ & $\tau_{2}\left(a_{2}\right)$ & $\chi^{2}$ \\
\hline Cyclohexane & 282 & 322 & 0.651 & 4405 & $725 \mathrm{ps}$ & - & $1 \cdot 8$ \\
\hline Dioxane & 291 & 335 & - & 4513 & - & - & - \\
\hline Chloroform & 289 & 334 & - & 4662 & - & - & - \\
\hline Acetonitrile & 287 & 338 & $0 \cdot 594$ & 5257 & $941 \mathrm{ps}$ & - & $1 \cdot 3$ \\
\hline DMSO & 300 & 348 & - & 4598 & 470 ps $(0 \cdot 12)$ & 1000 ps $(0 \cdot 88)$ & $1 \cdot 3$ \\
\hline Isopropanol & 296 & 349 & 0.088 & 5130 & $60 \mathrm{ps}(0 \cdot 33)$ & 248 ps $(0.67)$ & 0.9 \\
\hline Methanol & 295 & 353 & 0.086 & 5569 & & - & - \\
\hline Water & 286 & 362 & 0.042 & 7340 & \multicolumn{2}{|c|}{ Similar to IRF } & \\
\hline
\end{tabular}

$\phi$ is the quantum yield, $\tau$ is the lifetime value, $a_{1}, a_{2}$ represent corresponding relative amplitudes of different components in biexponential fitting of the temporal profile (decay monitored at emission maximum) 
shown in figure $1 \mathrm{~b}$, the emission spectra of AMBME is broad and largely red-shifted in cyclohexane. Similar Stokes-shifted emission bands for the CT state are also observed in 4-amino benzoic acid, 3amino benzoic acid and 2-amino benzoic acid. ${ }^{10-12}$ However, the Stokes shift of the emission band in AMBME is less than that of 2- and 3-amino benzoic acids indicating the lower polar character of AMBME compared to 2- and 3-amino benzoic acids. With increasing polarity of the solvent, i.e., going from cyclohexane to acetonitrile, a clear Stokes shift is observed in the emission band maximum. Dual fluorescence is observed in methanol and water. Hydrogen-bonding interaction with protic solvents may tune both the LE and CT energy states differently, thus we get distinct dual emission band in protic solvents. It is found that the position of the higher
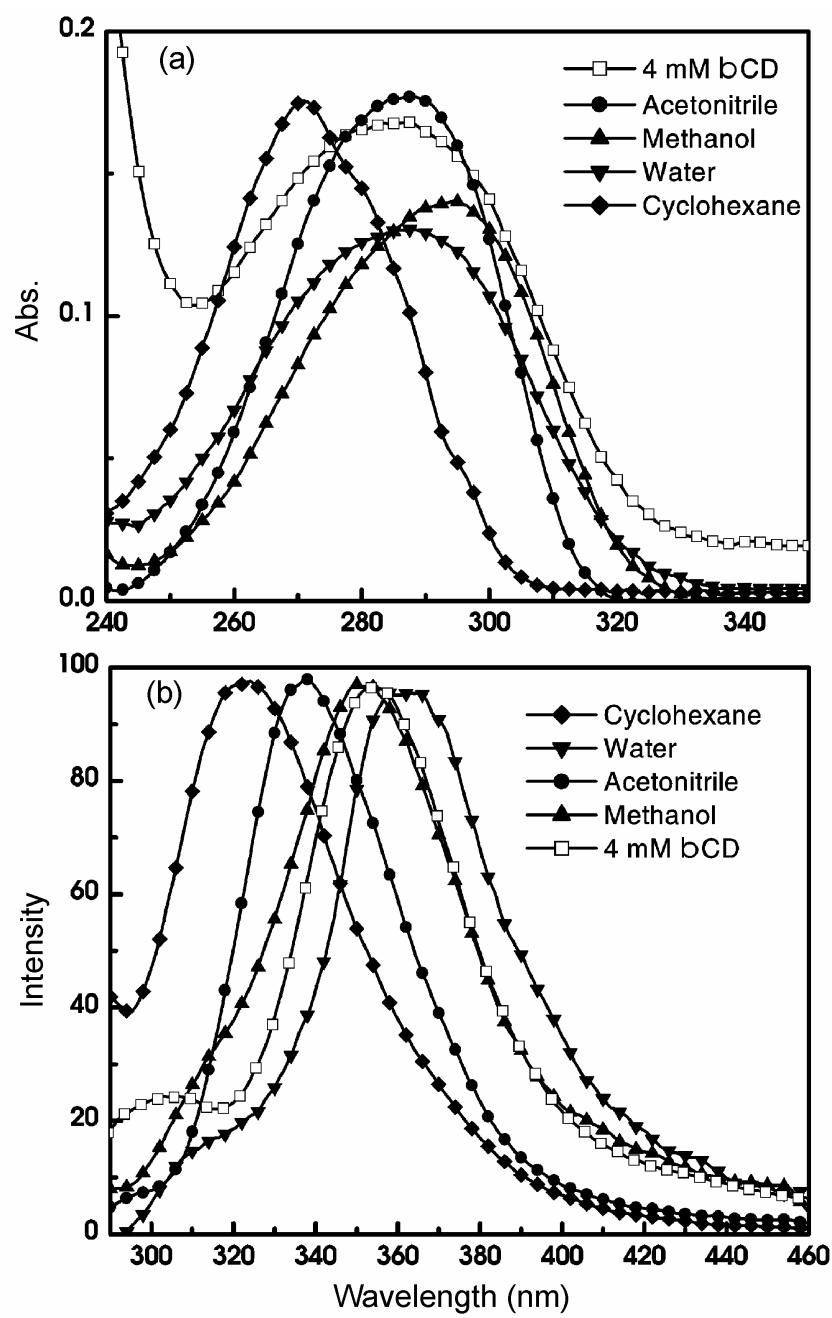

Figure 1. (a) Absorption, and (b) emission spectra of 4amino-3-methyl benzoic acid methyl ester $\left(\lambda_{\text {ext }}=280 \mathrm{~nm}\right)$. energy emission band at $\approx 310 \mathrm{~nm}$ is independent of solvent polarity, but the position of the lower energy band changes on changing the dielectric constant and hydrogen-bonding properties of the medium. The increase in Stokes shift of the low-energy emission indicates that this band arises from the CT state as was observed in other related molecules. ${ }^{10-12,31-33}$ It is to be noted that a distinct dual emission is observed in $\beta \mathrm{CD}(4 \mathrm{mM})$ solution which confirm the presence of both the LE and CT states (figure lb). The excitation spectra of both the emission bands are found to be independent of emission wavelength and agree reasonably well with the absorption spectrum. This indicates that CT emission is generated through LE state excitation. Almost linear dependency of the lower energy emission band maximum with solvent polarity parameter in the Lippert plot (figure 2a) again supports the charge-transfer nature of the excited state. Using the following Lippert equation, we have calculated the dipole moment of the excited CT state, ${ }^{31,34}$

$$
v_{a}-v_{f}=\left[2 / h c a^{3}\right] \Delta f\left[\mu^{*}-\mu\right]^{2}+\text { constant. }
$$

The term $\Delta f$ is known as solvent-polarity parameter and is related to dielectric constant and refractive index of the medium. In the above equation, $h, c, a$, $\mu$ and $\mu^{*}$ are Planck's constant, velocity of light, radius of the cavity in which the fluorophore resides, and ground and excited CT state dipole moments, respectively. The ground-state dipole moment $(\mu)$ and $a$ values were calculated using hybrid functional B3LYP and 6-311++G $(d, p)$ basis sets for the ground-state minimum energy structure. The calculated dipole moment for the ground state obtained from DFT calculation is $3.56 \mathrm{D}$. Dipole moment of the CT state obtained by solvatochromic method is $7 \cdot 14$ D. This clearly indicates that the high dipole moment of the CT state is responsible for the solvent-polarity-dependent red-shifted emission band.

Nonlinearity of the Lippert plot for the red-shifted band in protic solvents indicates that hydrogen bonding may play a significant role in determining the position of the low-energy emission band. As seen in figure $2 b$, the presence of two sets of straight lines in the plot of Stokes shift vs $E_{T}(30)$ parameter indicates that hydrogen bonding interaction is present along with the dipolar interaction. ${ }^{35}$ It is found that the lower energy emission band maximum of AMBME shows a linear correlation with hydrogen bonding parameter $\alpha$ of the solvent (figure 2c). ${ }^{36} \mathrm{~A}$ 
correlation is also observed between quantum yields and hydrogen bonding parameter $\alpha$. The decrease of fluorescence quantum yields with increase of hydrogen bonding tendency of the solvents indicates that
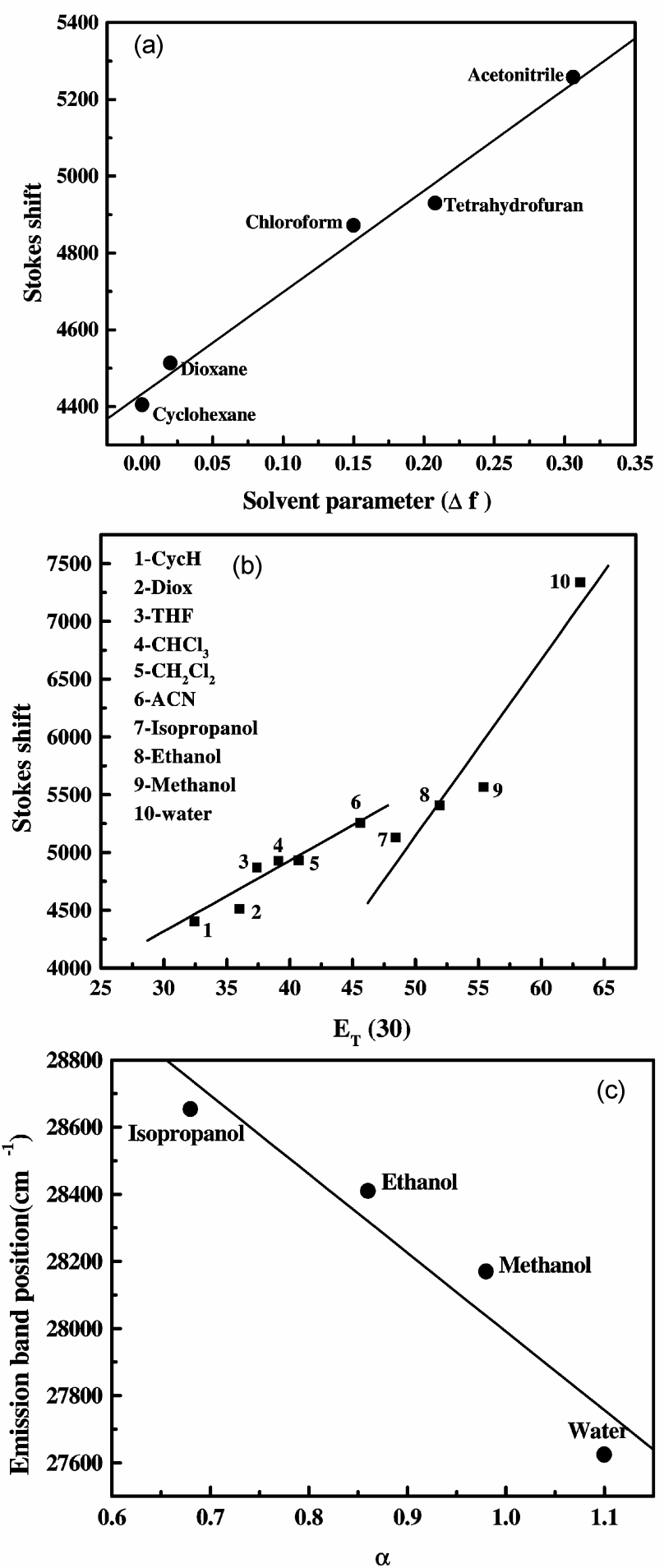

Figure 2. (a) Stokes shift $\left(\mathrm{cm}^{-1}\right)$ vs solvent parameter (Lippert plot); (b) Stokes shift $\left(\mathrm{cm}^{-1}\right)$ vs $E_{T} 30$ parameter; (c) fluorescence-band position of 4-amino-3-methyl benzoic acid methyl ester vs hydrogen-bonding parameter. hydrogen bonding can act as a non-radiative channel along the ICT path. ${ }^{37}$

Lifetime measurements data upon $280 \mathrm{~nm}$ excitation in different polarity solvents are shown in figure $3 \mathrm{a}$ and data are presented in table 1. Emission monitored at $325 \mathrm{~nm}$ in cyclohexane solvent is strictly single-exponential with decay time of $725 \mathrm{ps}$. In acetonitrile solvent, the fluorescence lifetime of $350 \mathrm{~nm}$ emission band is found to be $810 \mathrm{ps}$. Very close fluorescence lifetime values in acetonitrile and cyclohexane solvent indicate that the emission bands have the same origin. In DMSO solvent, the biexponential decay curve indicates the existence of both the LE and CT states. The lower fluorescence life-
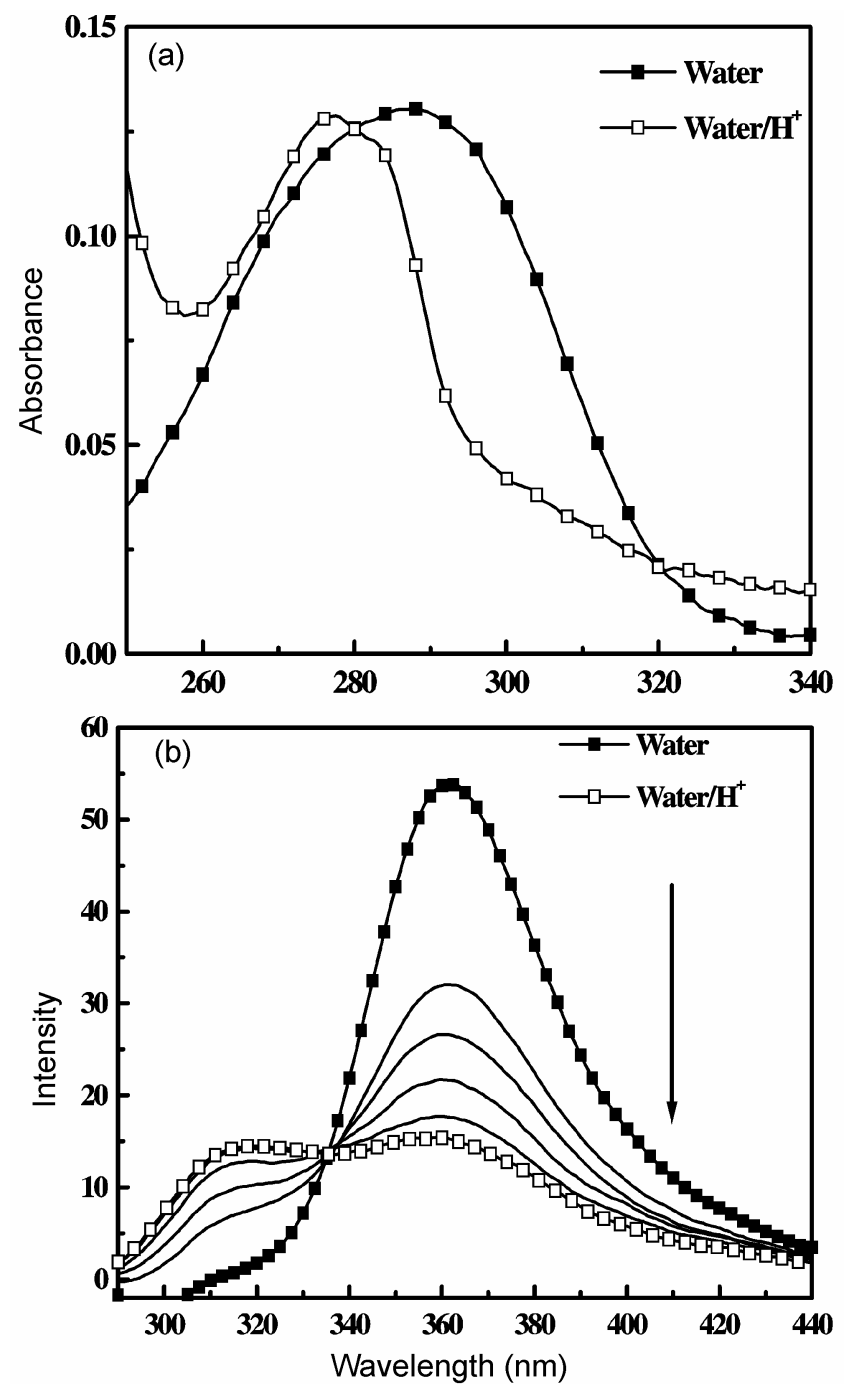

Figure 3. (a) Absorption and (b) emission spectra of 4amino-3-methyl benzoic acid methyl ester in presence of acid $\left(\lambda_{\text {ext }}=280 \mathrm{~nm}\right)$. Arrow indicates increase of acid concentration. 
time value of $470 \mathrm{ps}$ is assigned to the LE state and the higher value of $1000 \mathrm{ps}$ is assigned to the CT state. In isopropanol solvent both the 325 and $350 \mathrm{~nm}$ emission bands show biexponential decay curve with a fast decay of $\sim 60 \mathrm{ps}$ (minor component) and a slow decay of $\sim 248$ ps (major component). The low lifetime values again support the fact that hydrogen-bonding acts as a nonradiative channel in ICT path. The slower lifetime may be due to the hydrated CT state and the faster lifetime (which is faster than the instrument response function) may be due to the LE state of the hydrated clusters. In water the lifetime values are too fast to be able to detect under present conditions.

The change in the absorption and emission spectra of AMBME in presence of acid are shown in figures 3a,b. Adding of $\mathrm{H}^{+}$ion shows a small blue shift of the absorption spectra (figure $3 \mathrm{a}$ ) due to the protonated species formed by addition of $\mathrm{H}^{+}$ion to the lone pair of the nitrogen atom. However, a drastic change is observed in the emission spectra (figure $3 b)$. With increase of acid concentration the charge transfer band intensity decreases with the increase of LE emission band of the protonated species. As seen in figure $3 b$, the emission spectra in presence of acid indicate that both the CT band and protonated species coexist. This may be possible if some extent of the protonated species undergoes excited state deprotonation reaction. ${ }^{38}$ The excited deprototated state, i.e., the original neutral excited species immediately undergoes a transformation from LE to CT state in the excited state PES and hence shows CT band in addition to emission band of the protonated species.

The variation of emission intensity with temperature is shown in figure 4. As shown in figure 4a,b, the emission intensity decreases with increase of temperature. This indicates that temperature plays an active role as non-radiative decay channel in ICT path. The emission spectra (figure 4c) in ethanol glass matrix is found to be similar to that of $\mathrm{MCH}$ glass matrix and the blue shift of emission band in ethanol may reflect the change in solvent properties such as polarity, polarisibility and viscosity upon decreasing of temperature. ${ }^{30}$ The high viscosity in $77 \mathrm{~K}$ glass matrix also inhibits the relaxation process from LE to CT state through any twisting path, as a result we observed only the LE emission band in ethanol glass matrix.

The laser-induced fluorescence excitation spectrum of AMBME is shown in figure 5. The excita- tion spectrum shows a doublet feature with two strong bands at 34251 and $34266 \mathrm{~cm}^{-1}$ with some moderate and weak intense bands on the blue side of the intense band. The first pair of bands is separated
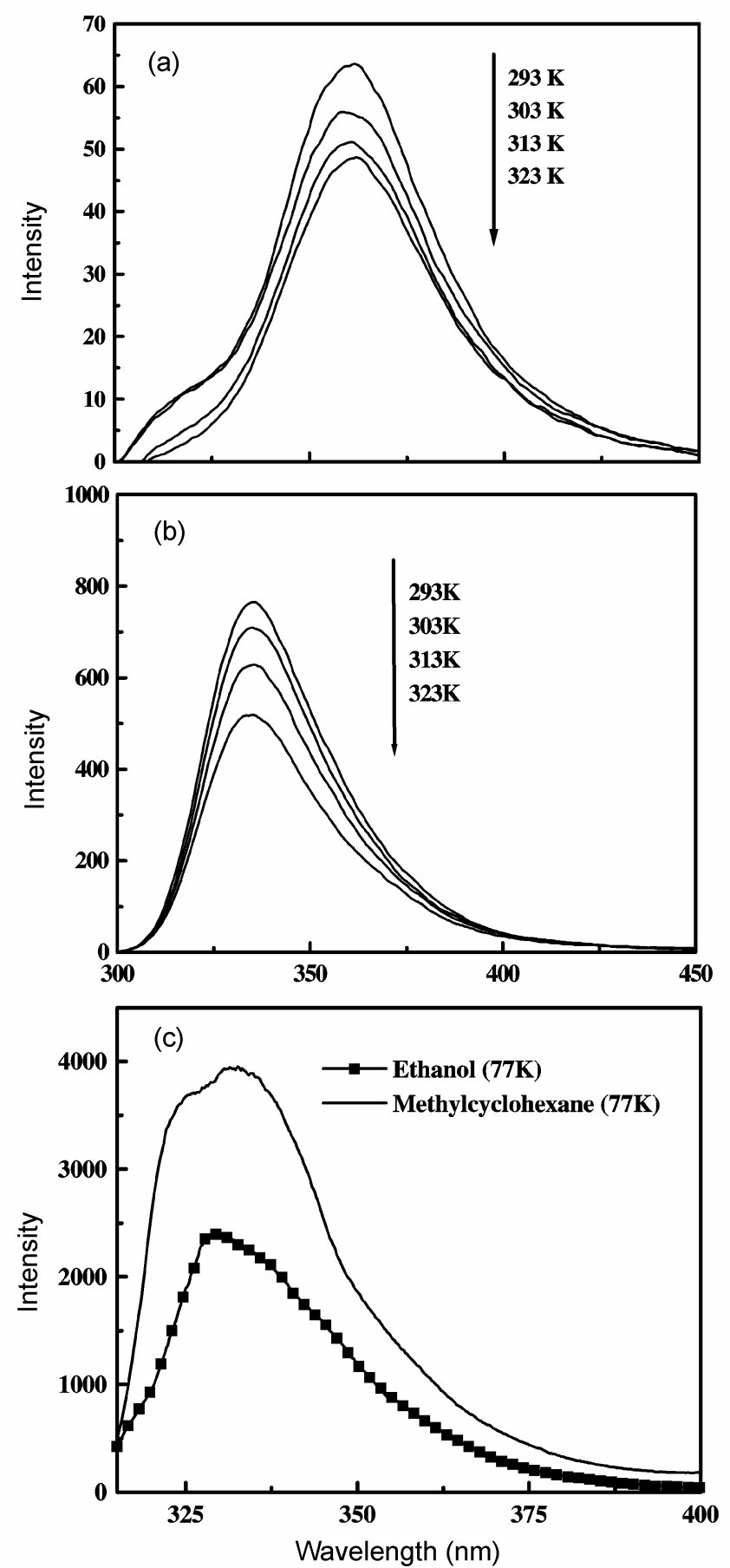

Figure 4. Temperature-dependent emission spectra of 4-amino-3-methyl benzoic acid methyl ester in (a) water, (b) acetonitrile, (c) ethanol and methylcyclohexane glass matrix at $77 \mathrm{~K}$. 
by only $15 \mathrm{~cm}^{-1}$. Interestingly, it seems that all other blue-sided bands appear as pairs. Apparently this type of spectral signature indicates that the first two bands correspond to the $0-0$ band of two species (i.e. two conformers) and the pairwise bands are similar types of low-frequency large-amplitude modes that ride over each of the $0-0$ band of two isomers. It is reported that $m$-aminobenzoic acid shows similar type of two $0-0$ band origins for the two conformers in the jet-cooled condition. ${ }^{21}$ The separation of the $0-0$ transition of two conformers of $m$-aminobenzoic acid is about $27 \mathrm{~cm}^{-1}$. Theoretically the molecule AMBME can form two low-energy structures where the $-\mathrm{CO}$ group of the ester moiety could be the same (conformer I) or opposite (conformer II) side of the ring methyl group (figure 6) and are very similar to that of $m$-aminobenzoic acid. The difference in energy between these two conformers in the ground state is found to be only $29 \mathrm{~cm}^{-1}$ (DFT/B3LYP/6-311++G $(d, p)$ ) and the barrier for interconversion between them is quite high $\left(2882 \mathrm{~cm}^{-1}\right)$. High barrier energy does not allow the freezing of molecules to only the lowest energy conformer in the cooled-jet. Theoretically it is expected that both the conformers should be present in the cooled jet. The difference in theoretical $S_{1} \leftarrow S_{0}$ vertical transition energy between two conformers is found to be about $19 \mathrm{~cm}^{-1}$ which corresponds well with the experimental $0-0$ transition energy difference of $15 \mathrm{~cm}^{-1}$. Therefore, we have assigned the doublet structure in the LIF excitation spectrum to

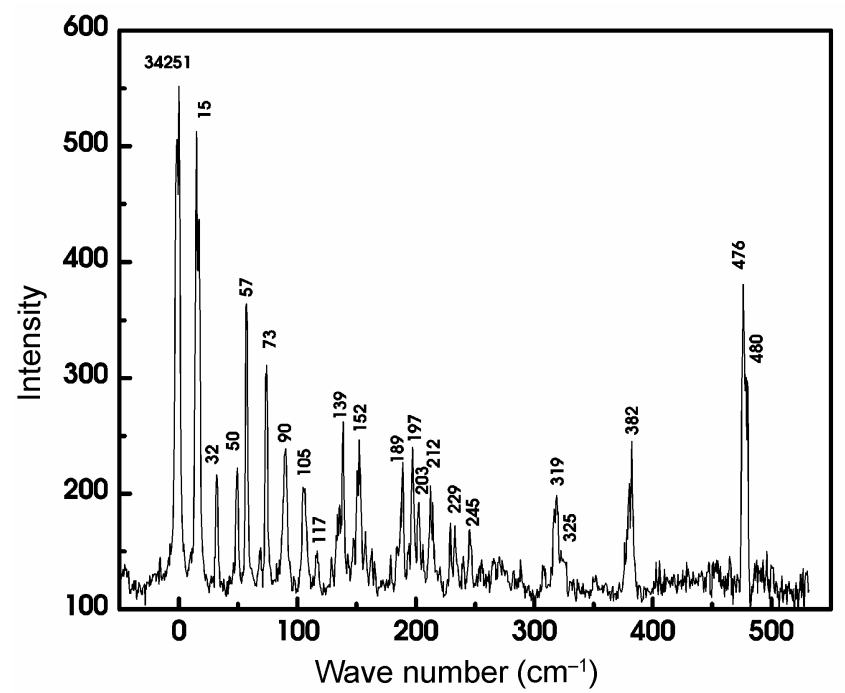

Figure 5. Laser-induced fluorescence excitation spectrum of 4-amino-3-methyl benzoic acid methyl ester in a cooled-molecular jet. two-origin transition of two low-energy conformers. However, further experiments, such as hole-burning measurement is needed to confirm this assignment. The assignment of the observed low-frequency modes is presented with the calculated normal modes for two conformers in table 2. It is found that several low-frequency large-amplitude modes of AMBME are similar in nature with that of its parent molecules $p$-amino- and $m$-aminobenzoic acid. ${ }^{21,22}$

We have tried to interpret dual fluorescence phenomenon of AMBME in the light of the TICT model using the density functional theoretical approach. Ground-state optimisation has been done using B3LYP hybride functional and 6-311++g $(d, p)$ basis. In the ground state conformers I and II are nonplanar and the dihedral angle between the $-\mathrm{NH}_{2}$ group and the benzene moiety is $\approx 19^{\circ}$. Usually these pre-twisted compounds are known to emit preferentially or only show CT emission in polar solvent. ${ }^{39,40}$ As the optimised geometry is nonplanar, no symmetry element could be applied for the various states. We have used the minimum energy structure (conformer I) for calculating spectroscopic properties of the molecule, since the two possible conformers are identical in nature and it is expected that the nature of condensed phase broad spectra would be same for both conformers. Calculated spectroscopic parameters are presented in table 3. Experimental results showed that the molecule AMBME shows absorption bands at $282 \mathrm{~nm}(4.39 \mathrm{eV})$ in cyclohexane and at $287 \mathrm{~nm}(4.31 \mathrm{eV})$ in acetonitrile. Our calculations

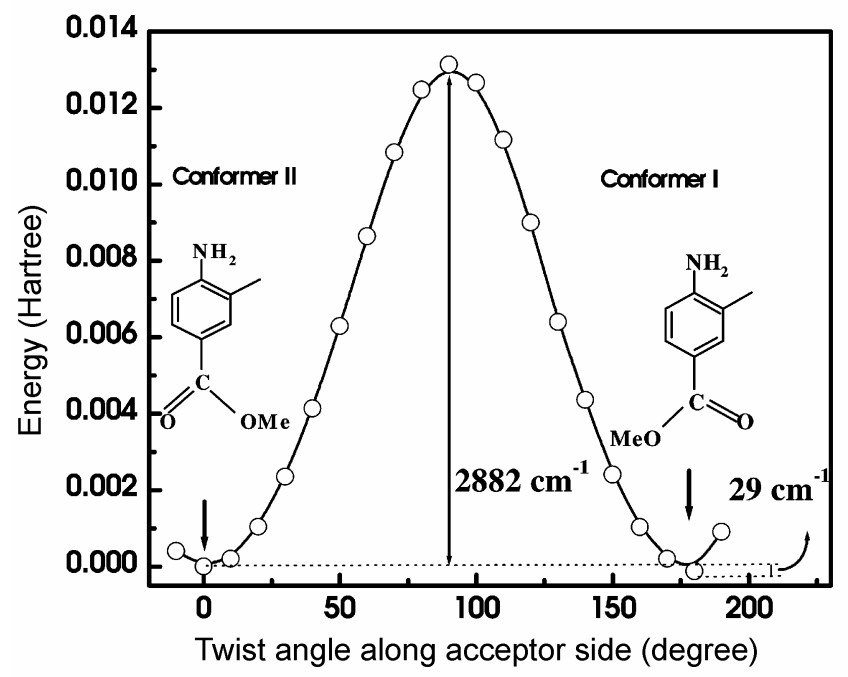

Figure 6. Calculated low-energy conformers I and II, and the potential energy curve obtained for torsional motion of ester group using B3LYP hybride functional and $6-311++\mathrm{g}(d, p)$ basis set. 
Table 2. Main excited vibronic bands of 4-amino-3-methyl benzoic acid methyl ester and their tentative assignment.

\begin{tabular}{|c|c|c|c|c|}
\hline \multicolumn{2}{|c|}{ Conformer I } & \multicolumn{2}{|c|}{ Conformer II } & \multirow[b]{2}{*}{ Assignment of calculated modes } \\
\hline Experimental $^{\mathrm{a}}$ & Calculated $^{\mathrm{b}}$ & Experimental $^{\mathrm{c}}$ & Calculated $^{\mathrm{b}}$ & \\
\hline 32 & 52 & 35 & 55 & Ester group torsion coupled to $\mathrm{CH}_{3} / \mathrm{NH}_{2}$ torsion \\
\hline 57 & 84 & 58 & 84 & Ester group torsion coupled to $\mathrm{CH}_{3} / \mathrm{NH}_{2}$ torsion \\
\hline 90 & 120 & 90 & 129 & Me group ester rotation \\
\hline 139 & 137 & 137 & 135 & Ester in-plane bending \\
\hline $189^{\mathrm{c}}$ & 156 & 182 & 154 & $\begin{array}{l}\text { Methyl of ester torsion coupled to ring out-of } \\
\text { plane motion }\end{array}$ \\
\hline 203 & 177 & 197 & 177 & Ring - Me rotation (pure) \\
\hline $229^{\mathrm{c}}$ & 202 & 230 & 202 & $\begin{array}{l}\mathrm{NH}_{2} \text { and methyl torsion coupled to out-of plane } \\
\text { ester }\end{array}$ \\
\hline $319^{\mathrm{c}}$ & 334 & 310 & 335 & Ring breathing \\
\hline $382^{\mathrm{c}}$ & 449 & & 447 & Ring out-of plane \\
\hline $476^{\mathrm{c}}$ & 485 & 465 & 486 & $-\mathrm{NH}_{2}$ inversion (pure) \\
\hline
\end{tabular}

${ }^{\mathrm{a}}$ With respect to $34251 \mathrm{~cm}^{-1}$; ${ }^{\mathrm{b}}$ calculation at DFT level (B3LYP $/ 6-311++\mathrm{G}(d, p)$, ${ }^{\mathrm{c}}$ with respect to $34266 \mathrm{~cm}^{-1}$; ${ }^{\mathrm{c}}$ similar bands for $m$-amino- and $p$-aminobenzoic acid ${ }^{21,22}$

Table 3. Computed parameters of 4-amino-3-methyl benzoic acid methyl ester in vacuum and acetonitrile solvent using DFT method with B3LYP hybride functional and 6-311++g $(d, p)$ basis set.

\begin{tabular}{lcccccccc}
\hline \multirow{2}{*}{ Medium } & & \multicolumn{2}{c}{ Absorption } & & \multicolumn{3}{c}{ Emission } \\
\cline { 3 - 4 } \cline { 7 - 8 } & State & $E_{\mathrm{th}}(\mathrm{eV})$ & $E_{\mathrm{ex}}(\mathrm{eV})$ & & $E_{\mathrm{th}}{ }^{\mathrm{a}}(\mathrm{eV})$ & & $E_{\mathrm{th}}{ }^{\mathrm{b}}(\mathrm{eV})$ & $E_{\mathrm{ex}}(\mathrm{eV})$ \\
\hline Vacuum & $S_{1}$ & 4.59 & 4.39 & & 4.32 & 4.33 & 3.85 \\
Acetonitrile & $S_{1}$ & 4.37 & 4.31 & & 4.08 & 3.98 & 3.66 \\
\hline
\end{tabular}

${ }^{a}$ Emission energy due to rotation of donor group towards the aromatic methyl group; ${ }^{b}$ emission energy due to rotation of donor group opposite two the aromatic methyl group; $E_{\text {th }}$ is the calculated energy value $\left(E_{\text {excited }}-E_{\text {ground }}\right)$ at DFT level (B3LYP/6-311++G $\left.(d, p)\right) . E_{\text {ex }}$ is the experimental value

show that the $S_{1} \leftarrow S_{0}$ transition energy is $270 \mathrm{~nm}$ $(4.59 \mathrm{eV})$ and $283.6 \mathrm{~nm}(4.37 \mathrm{eV})$ in vacuum and in acetonitrile solvent, respectively (we may consider that the calculation in vacuo is almost equivalent to that in a nonpolar solvent). Hence, the deviation of transition energy between the experimental and theoretical values in cyclohexane is $0.20 \mathrm{eV}$ and in acetonitrile is $0.06 \mathrm{eV}$. Comparing the absorption spectra of AMBME with other similar compound we assume that the broadness of the absorption band arises due the overlapping of two low-lying energy states. Calculations by TDDFT-PCM (B3LYP/6$311++\mathrm{g}(d, p))$ level also show that the energy difference between $S_{1}$ and $S_{2}$ state is only $\approx 0.27 \mathrm{eV}$ even in acetonitrile solvent. Therefore, overlapping of two excited states is highly possible even in polar solvent. For the twisted conformer, the calculated transition energy in acetonitrile solvent shows a red shift relative to the gas phase. The red shift is about $0.29 \mathrm{eV}$ and $0.39 \mathrm{eV}$ in acetonitrile due to rotation of donor group towards and opposite to the aromatic methyl group, respectively (table 3 ). This shifting is not sufficient as expected from the large difference between the static dielectric constant of acetonitrile (36.64) and cyclohexane (2.04). The experimental fluorescence maximum appears at $322 \mathrm{~nm}(3.85 \mathrm{eV})$ and $338 \mathrm{~nm}(3.66 \mathrm{eV})$ in cyclohexane and acetonitrile, respectively. For twisting of the donor side i.e. $-\mathrm{NH}_{2}$ group (towards the substituted methyl group and also in the opposite direction) a possible redshifted emission is observed in both the directions of rotation. This red shift is more for the rotation towards the opposite side of substituted methyl group due to less steric hindrance. In acetonitrile solvent, the calculated red-shifted emission energies are $3.98 \mathrm{eV}$ and $4.08 \mathrm{eV}$ (rotation opposite to methyl group and towards the methyl group, respectively). It is observed that in either case calculated values are higher than the experimental findings.

The theoretical ground and excited state potential energy surfaces and the oscillator strength and transition energy value along the twisting coordinate are shown in figure 7 . The trend in calculated results in vасиио is the same as in the acetonitrile solvent. The excited state PES shows an asymmetric double-well potential. The potential energy surface along the 
twist coordinate at the donor side in both the direction shows that the CT state with perpendicular geometry of the nitrogen lone pair is higher than the LE state even in acetonitrile solvent. However, lowering of vertical transition energy and oscillator strength value (figure $7 \mathrm{~b}$ ) along the donor twist coordinate indicate red-shifted emission and $n-\pi^{*}$ character of the emitting species, respectively. Molecular orbital picture of AMBME in its global minima state shows that the HOMO-LUMO transition (figure $8 \mathrm{a}, \mathrm{b}$ ) is of $\pi-\pi^{*}$ nature and donor lone pair is delocalised. Whereas, for both of the twisted conformer (due to rotation of donor group in different direction) the HOMO-LUMO transition (figure 8c-f) is of $n-\pi^{*}$ character and the donor lone pair is localized and available for charge transfer to the acceptor side. This indicates that charge transfer takes place when decoupling is maximum between the
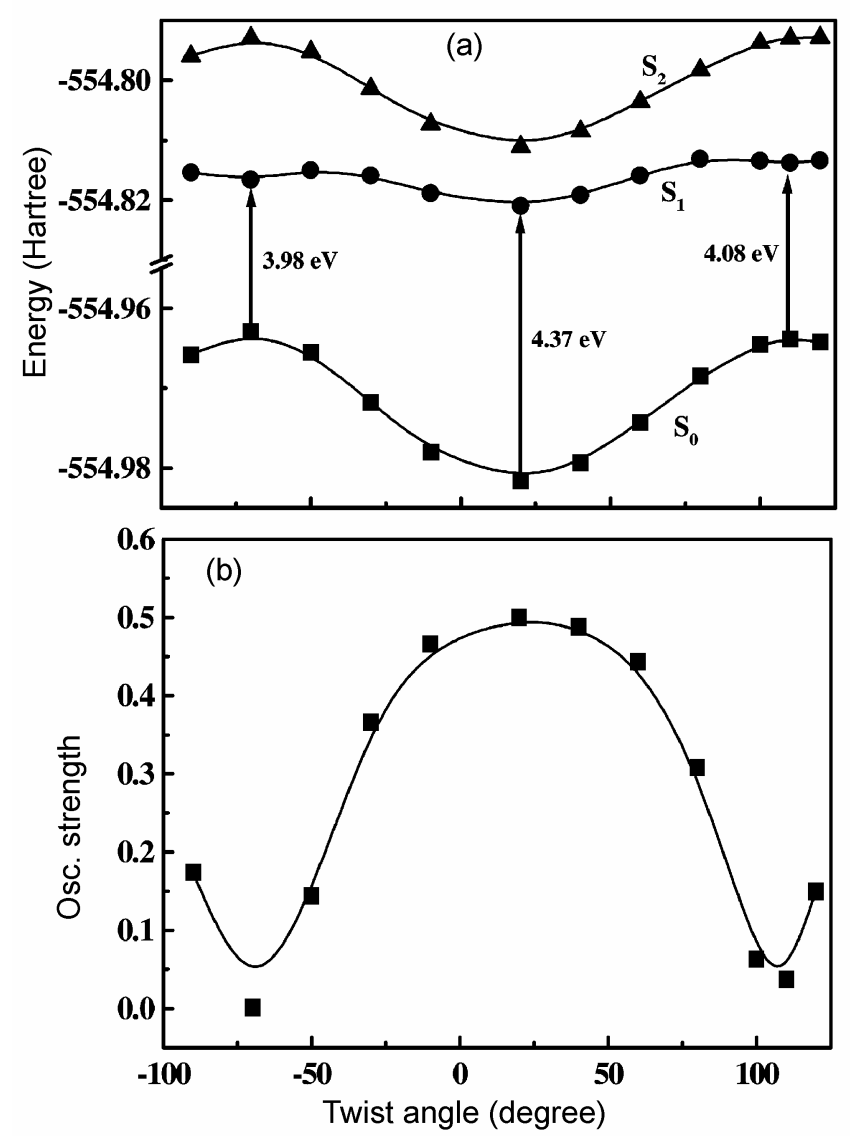

Figure 7. Potential energy surface (PCM-TDDFT model with $6-311++\mathrm{G}(d, p)$ basis set) of the ground and first two excited states along the twisting coordinate of donor group in acetonitrile solvent, (b) plot of oscillator strength of $S_{1}$ state along the twisting coordinate of the donor part. donor and acceptor group, i.e. donor group become orthogonal to the acceptor moiety. The weak donor ( $-\mathrm{NH}_{2}$ group) may be responsible for this type of high lying CT state in the PES. Similar type potential energy surfaces are also obtained in some thiophene-containing biaryl systems, ${ }^{41}$ where it is considered that weak donor-acceptor combination is responsible for this type of PES. Theoretically it is already reported that introducing better donor groups like $-\mathrm{NMe}_{2}$ in place of $-\mathrm{NH}_{2}$ group lower the CT state below the LE state in the excited state. ${ }^{28}$ Calculations with the rotation of the acceptor group do not show any double minima type of potential and lowering of vertical transition energy (figure not given).

Another possible explanation of the discrepancy between the calculated potential energy surface and the experimental results could be attributed to excited state geometry without full optimisation. This type of excited state optimisation calculation is beyond our scope as analytical gradients are not available for the TDDFT method. Structurally it is obvious that $-\mathrm{NH}_{2}$ rotation should be influenced by the ortho methyl group due to some steric effect. Hence, incorporation of $-\mathrm{CH}_{3}$ group motion with donor torsion may improve the calculation towards the experimental findings. (a)

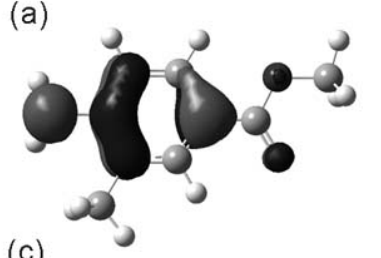

(c)

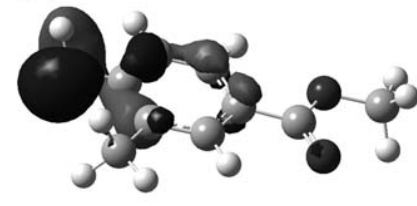

(e)

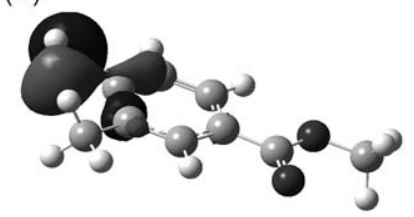

(b)

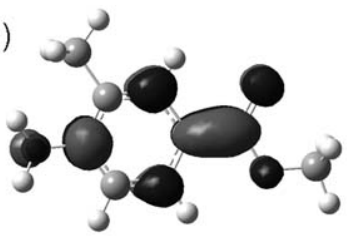

(d)

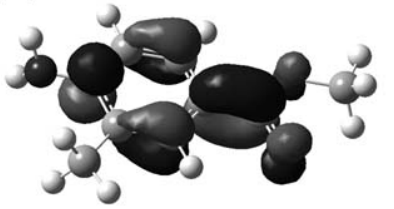

(f)

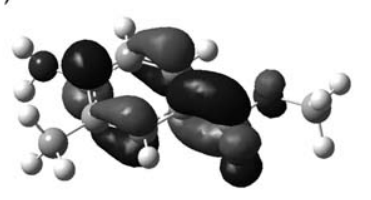

Figure 8. (a) HOMO and (b) LUMO of ground state; (c) HOMO and (d) LUMO of twisted ground state (twisting of donor opposite to methyl group); (e) HOMO and (f) LUMO of twisted ground state (twisting of donor towards methyl group). 


\section{Conclusions}

The fluorescence spectroscopy of AMBME has been studied in condensed phase and in jet-cooled molecular beams in combination with quantum chemical calculations. In the condensed phase, the molecule shows red-shifted emission in solvents of difficult polarities. The charge transfer state is characterized by higher excited state dipole moment obtained from solvatochromic measurements. A clear dual fluorescence is observed in protic solvents where the high energy band corresponds to the hydrogen-bonded LE state and low-energy band to the hydrogenbonded CT state. The jet-cooled condition the molecule present as two low-energy structures with respect to the orientation of the acceptor group. The LIF spectrum shows good correlation with respect to two conformers at the low frequency region. Calculations using TDDFT and PCM-TDDFT methods predict an asymmetrical double-well potential in the excited state and a red-shifted emission from the twisted CT state.

\section{Acknowledgement}

This work was supported by a grant from Department of Science and Technology. The authors thank Dr P K Gupta and Dr Kaustuv Das of CAT, Indore for providing help with and use of the fluorescence lifetime measurement facility. The authors also thank Professor Tapan Ganguly, Department of Spectroscopy, for low temperature measurements.

\section{References}

1. Lippert E, Luder W and Boos H 1962 Advances in molecular spectroscopy (ed.) A Mangini (Oxford: Pergamon) p. 443

2. Grabowski Z, Rotkiewicz K and Rettig W 2003 Chem. Rev. 1033899

3. Rettig W 1994 Top. Curr. Chem. 169253

4. Rotkiewicz K, Grellmann K H and Grabowski Z R 1973 Chem. Phys. Lett. 19315

5. Zachariasse K A 2000 Chem. Phys. Lett. 8320

6. Sobolewski A L, Sudholt W and Domcke W 1998 J. Phys. Chem. A102 2716

7. Serrano-Andres L, Merchan M, Roos B O and Lindh $\mathrm{R} 1995 \mathrm{~J}$. Am. Chem. Soc. 1173189

8. Zachariasse K A, von der Haar T, Hebecker A, Leinhos U and Kuhnle W 1993 Pure Appl. Chem. 651745

9. Galievsky V A, Druzhinin S I, Demeter A, Jiang YB, Kovalenk S A, Lustres L P, Venugopal K, Ernsting N P, Allonas X, Noltemeyer M, Machinek R and Zachariasse K A 2005 Chem. Phys. Chem. 62307
10. Stalin T, Shanthi B, Vasantha Rani P and Rajendiran N 2006 J. Inclusion Phenomenon Macrocyclic Chem. 5521

11. Stalin T and Rajendiran N 2006 Chem. Phys. 322311

12. Stalin $\mathrm{T}$ and Rajendiran N $2006 \mathrm{~J}$. Photochem. Photobiol. A182 137

13. Daum R, Druzhinin S, Ernst D, Rupp L, Schroeder J and Zachariasse K A 2001 Chem. Phys. Lett. 341272

14. Bli $\beta$ B, Lommatzsch U, Monte C, Rettig W and Brutschy B 2000 Chem. Phys. 254407

15. Saigusa H, Iwase E and Nishimura M 2003 J. Phys. Chem. A107 4989

16. Gibson E M, Jones A C and Phillips D 1987 Chem. Phys. Lett. 136454

17. Pereira R, Alava I and. Castano F 1994 J. Chem. Soc., Faraday Trans. 902443

18. Howells B D, McCombie J, Frank Palmer T, Simon J $\mathrm{P}$ and Walter A 1992 J. Chem. Soc., Faraday Trans. 882595

19. Fernandez J A, Longarte A, Unamuno I and Gastauo F $2000 \mathrm{~J}$. Chem. Phys. 1135804

20. He Y, Wu C and Kong W 2005 J. Phys. Chem. 109 2809

21. He Y, Wu C and Kong W 2004 J. Chem. Phys. 1213533

22. He Y, Wu C and Kong W 2005 J. Chem. Phys. 1218321

23. Das K, Jain B and Patel H S 2006 J. Phys. Chem. A110 1698

24. Frisch M J et al 2003 Gaussian 03, Revision B.03, Gaussian, Inc., Pittsburgh PA

25. Duan X-H, Li X-Y, He R-X and Cheng X-M $2005 \mathrm{~J}$. Chem. Phys. 122084314

26. Cammi R, Mennucci B and Tomasi J 2000 J. Phys. Chem. A104 5631

27. Jodicke C J and Luthi H-P 2002 J. Chem. Phys. 117 4146

28. Jodicke C J and Luthi H-P 2002 J. Chem. Phys. 117 4157

29. Jodicke C J and Luthi H-P 2003 J. Chem. Phys. 119 12852

30. Jodicke C J and Luthi H-P 2003 J. Am. Chem. Soc. 125 252

31. Chakraborty A, Kar S and Guchhait N 2006 Chem. Phys. 32075

32. Bangal P R and Chakraborty S 1998 J. Photochem. Photobiol. A116 191

33. Bangal P R, Panja S and Chakraborty S $2001 \mathrm{~J}$. Photochem. Photobiol. A139 5

34. Lewis F D and Weigel W 2000 J. Phys. Chem. A104 8146

35. Subuddhi U, Haldar S, Sankararaman S and Mishra A K 2006 Photochem. Photobiol. Sci. 5459

36. Taft R W and Kamlet M J 1976 J. Am. Chem. Soc. 98 2886

37. Testa A C 1991 J. Lumin. 50243

38. Sumalekshmy S and Gopidas K R 2004 J. Phys. Chem. B108 3105

39. Grabowski Z R, Rotkiewicz R, Siemiarczuk A, Cowley D J and Baumann W 1979 Nouv. J. Chem. 3443

40. Rettig W and Zietz B 2000 Chem. Phys. Lett. 317187

41. Rettig W, Kharlanov V, Effenberger F and Steybe F 2005 Chem. Phys. Lett. 404272 
